\title{
Comparison between the effect of kartogenin and TGF $\beta 3$ on chondrogenesis of human adipose- derived stem cells in fibrin scaffold
}

\author{
Valiani A, Izadi MA, Bahramian H, Esfandiari E, Hashemibeni B \\ Department of Anatomical Sciences and Molecular Biology, Medical School, Isfahan University \\ of Medical Sciences, Isfahan, Iran. hashemibeni@med.mui.ac.ir
}

\begin{abstract}
BACKGROUND: Due to very sluggish turnover at the molecular and cellular level, the healing of chondral damages has been considered difficult. In the current study, the effects of the Kartogenin, a small heterocyclic molecule on chondrogenic differentiation of stem cells was compared to TGF- $\beta 3$.

METHODS: Human Adipose-Derived Stem Cells were extracted during an elective surgery. Cell viability was estimated by MTT assay, differentiated cells evaluated by histological and immunohistochemical techniques. Expression of cartilage specific genes (SOX9, Aggrecan, type II and X collagens) assessed by real-time PCR. RESULTS: The real-time PCR assay has revealed the expression of gene marker of chondrogenesis, SOX9, Aggrecan and type II collagen, both in Kartogenin and TGF $\beta 3$ groups compared to the control group, significantly $(p<0.05)$. A low expression level of collagen type $X$ as a hypertrophic marker was seen in cartilage produced by using Kartogenin. Meanwhile, the level of type $X$ collagen protein in Kartogenin group was significantly decreased ( $p>0.05)$ compared to TGF- $\beta 3$ group.

CONCLUSION: Kartogenin was suitable for successful chondrogenic differentiation of human adipose- derived stem cells and a suppressor of the consequent hypertrophy (Tab. 1, Fig. 5, Ref. 31). Text in PDF www.elis.sk. KEY WORDS: kartogenin, TGF 3 , fibrin scaffold, chondrogenesis, adipose-derived stem cells.
\end{abstract}

\begin{abstract}
Abbreviations
KGN - Kartogenin, TGF 33 - transforming growth factor beta3, ADSCs - adipose-derived stem cells, MTT - 3-(4,5-Dimethylthiazol-2-Y1)-2,5-Diphenyltetrazolium Bromide, SOX9 - sex determining region Y-box 9, COLII - Collagen type II,COLX - Collagen type X,AGG - Aggrecan, MSCs - mesenchymal stem cells, hMSCs - Human Mesenchymal Stem Cells, N-CAM - Neural Cell Adhesion Molecule, nmol - Nano mole, RT-PCR - Reverse transcriptase- polymerase chain reaction, mRNA - Messenger Ribonucleic Acid, FLNA - Filamin A, CBFb - Core-Binding Factor, Beta, RUNX - Runt-related protein, PBS - Phosphate Buffered Saline, FBS - Foetal bovine serum, DMEM - Dulbecco's Modified Eagle's Medium, FFP - Fresh frozen plasma, BSA - Bovine Serum Albumin, cDNA - complementary DNA, H\&E - Hematoxylin and eosin, IHC - Immunohistochemistry.
\end{abstract}

Department of Anatomical Sciences and Molecular Biology, Medical School, Isfahan University of Medical Sciences, Isfahan, I.R. Iran

Address for correspondence: $\mathrm{B}$. Hashemibeni, PhD, Department of Anatomical Sciences and Molecular Biology, Medical School, Isfahan University of Medical Sciences, I.R. Iran, Post Code: 81744-176, Isfahan, Islamic Republic of Iran. Phone: +98.311.7922426, Fax: +98.311.7922517

Acknowledgements: This work was supported by Deputy of Research of Isfahan University of Medical Sciences grant. This study was funded by a Grant (No. 395175) from the Research Council of the Medical University of Isfahan, Isfahan, Iran.

\section{Introduction}

Due to very slow turnover at the molecular and cellular levels and the low renovating capacity of articular chondrocyte, the healing of chondral damages was considered tremendously difficult(1). Globally, about $10 \%$ of elderly men and $20 \%$ of women over 65 years have signs of osteoarthritis (2). Today, tissue engineering can be valuable to improve satisfying methods for osteoarthritis (3). Requirements of constant progress in tissue engineering strategies are related to stem cells with proper scaffolds and chondrogenic signals (3). Human adipose tissue is considered as a good candidate for obtaining adult stem cells for regenerative medicine, because they can be acquired simply in great numbers and can be achieved through less invasive trial. In addition, it has been shown that adipose tissue includes many cells that are capable of differentiation into a variety of cell forms, compared with a few cells that have this potential in bone marrow $(4,5)$. Furthermore, easy procedures for obtaining them and their capacity for multipotent differentiation are helpful for consideration as a source of cells for the current of cartilage damages. Human adipose-derived stem cells (HADSCs) can simply be expanded in the culture for numerous passages to achieve an adequate and uniform population of cells before differentiating into chondrocytes. On the other hand, the characteristics caused by this differentiation and ability of consecutively passaged HADSc have not been reported in details, 


\section{1-597}

yet (4). Studies showed that the scaffolds used for cartilage tissue engineering as a $3 \mathrm{D}$ environment, aid to conserve contact properties more than monolayer culture. The features of a scaffold consists of biocompatibility, mechanical strengths, biodegradability, porosity and toxicity (5). The scaffold must be structurally strong, let cells infiltrate and is also used for growth factors distribution. These properties of scaffolds provide a healthy microenvironment for cells, suitable cell-cell relations and proper nutrient exchange in vitro (5). Fibrin is an accepted component with a high potential to use in tissue engineering. Besides, it can be obtained from the patient's own blood to be used as an autologous scaffold to decline the potential dangers of immunological response or contaminations $(6,7)$. In vivo, fibrin has an important role in inflammation, wound restorative, homeostasis and angiogenesis. Furthermore, in interaction with fibrin, cells will more and more replace the fibrin scaffold by their specific extracellular matrix (8). Chondrogenic differentiation of mesenchymal stem cells would be induced by several extrinsic and intrinsic elements. In this procedure, growth factors had the most significant effects (9). Growth factors control integrity and homeostasis in the hyaline cartilage, in addition to development (10). Transforming growth factor- $\beta 3$ (TGF- $\beta 3$ ) is a factor that plays a strategic role in cartilage development (11). The effect of TGF- $\beta 3$ is in the principal happenings in chondrogenesis, such as: chondrogenic condensation by motivation of N-CAM regulation and fibronectin synthesis (12), chondroprogenitor cell generation and differentiation via stimulation of SOX9 transcription (13). Despite the effects above, it has been revealed that TGF- $\beta 3$ has undesirable effects on cartilage. Many studies presented that in normal murine joints, overexpression of TGF- $\beta 3$ is associated with a stimulation of osteophyte, inflammation, outgrowth or fibroplasia of synovial liquid (14). Consequently, obtaining the best combination of a growth factor is the ideal aim of tissue engineering. From many structurally different, heterocyclic, small molecules separated, one composite, which is named Kartogenin (KGN), facilitates chondrocyte differentiation from human mesenchymal stem cells (HMSCs) in a dose-dependent method (median effective concentration $($ EC50) $=100 \mathrm{nmol})(15)$. Lineage-specific differentiation in the presence of KGN was further confirmed by fluorescent immunostaining of specific proteins, including type II collagen, SOX9, and aggrecan under both monolayer and highdensity conditions. Reverse transcriptase- polymerase chain reaction (RT-PCR) with mRNA isolated from differentiated cells established the expressions for example: superficial zone protein (SZP), aggrecan and type II collagen (16). KGN role is binding FLNA and disrupting its relations with $\mathrm{CBFb}$, which in turn controls the RUNX family of transcription factors( 17). Nowadays, small drug-like molecules carefully regulate transcription factor subcellular localization and downstream transcriptional activities. Thus, small molecules seem to be suitable tools for a selective modulation of gene transcription in cells, complete organisms, and in this case, have provided new visions into chondrocyte biology that may lead to novel treatments for this degenerative disease. In the current study, we investigated the effect of KGN in comparison to TGF- $\beta 3$ on human adipose-derived stem cell. Furthermore, in this article, the chondrogenic effect of KGN was examined.

\section{Materials and methods}

\section{Isolation and culture of human ADSCs}

Adipose tissue was collected from patients by elective surgery and under sterilized condition transferred to the laboratory. The consent was achieved from each patient previously. At that moment, adipose tissue was washed with PBS (Sigma-Aldrich) three times. Then, it was digested with $1 \mathrm{mg} / \mathrm{ml}$ collagenase type I (Sigma-Aldrich) at $37{ }^{\circ} \mathrm{C}$ for $45 \sim 60 \mathrm{~min}$. After that, the cell solution was centrifuged at $1700 \mathrm{RPM}$ for $8 \mathrm{~min}$. At the termination, the supernatant was removed and the resultant pellet was suspended in the medium contained DMEM complemented with $100 \mathrm{U} / \mathrm{ml}$ penicillin/streptomycin, $10 \% \mathrm{FBS}$ and then cultivated in a humidified atmosphere of $95 \%$ air and $5 \% \mathrm{CO}_{2}$ at $37{ }^{\circ} \mathrm{C}$. The medium was changed after 24 hours and every 3 days. When the cells reached $95 \%$ confluence at the third passage (Fig. 1) it was collected with $0.25 \%$ trypsin and used at a concentration of $1 \times 10^{6}$ cells for each scaffold (18).

\section{Preparation of fibrinogen and thrombin}

Fresh frozen plasma solutions (FFP) and fibrinogen were obtained from The Isfahan Blood Transfusion Organization. FFP was defrosted in a water bath at $37^{\circ} \mathrm{C}$ and then $15 \mathrm{ml}$ of it was mixed with $10 \mathrm{ml}$ of gluconate calcium. The acquired solution was incubated for $3 \mathrm{~h}$ at $37^{\circ} \mathrm{C}$ and then centrifuged at $2500 \mathrm{RPM}$ for $10 \mathrm{~min}$. The supernatant was harvested as thrombin. Fibrinogen and thrombin solutions were prepared for use as a cell culture.

\section{In vitro chondrogenic differentiation}

The adipose tissue-derived stem cells at a concentration of $1 \times 10^{6}$ cells were liquified within fibrinogen and thrombin with the equal amount $(350 \mu \mathrm{L})$ was added to them. At that time, the fibrin scaffolds in chondrogenic medium containing DMEM-high glucose along with $1 \%$ insulin-transferrin-selenium (Sigma-Aldrich), $50 \mu \mathrm{g} / \mathrm{ml}$ ascorbate 2-phosphate, dexamethasone $100 \mathrm{nmol}$ (Sigma-Aldrich), $50 \mathrm{mg} / \mathrm{ml} \mathrm{BSA,} 5 \mu \mathrm{g} / \mathrm{ml}$ linoleic acid (SigmaAldrich), $1 \%$ penicillin/streptomycin without any growth factor as the control group and chondrogenic medium added $100 \mathrm{nmol}$ KGN (Sigma-Aldrich) and $10 \mathrm{ng} / \mathrm{ml} \mathrm{TGF} \beta 3$ as the treatment groups were located in the incubator $\left(37^{\circ} \mathrm{C}, 5 \% \mathrm{CO}_{2}, 99 \% \mathrm{hu}-\right.$ midity) for 14 days. Then $500 \mu \mathrm{ml}$ of the chondrogenic medium was added to each well. The half amount of medium was substituted every 3 days.

\section{Cell viability assay}

HADSc were seeded within the fibrin scaffolds in chondrogenic medium and were incubated for 14 days $\left(37^{\circ} \mathrm{C}, 5 \% \mathrm{CO}_{2}\right.$, $99 \%$ humidity). Cells viability and proliferation were evaluated by MTT (3-(4,5-dimethyl) thiazol-2-yl-2,5-dimethyl tetrazolium bromide, $5 \mathrm{mg} / \mathrm{ml})$. Compound MTT was added per well after eliminating chondrogenic medium and incubated at $37^{\circ} \mathrm{C}$ for $4 \mathrm{~h}$. Then, the medium was eliminated and intracellular formazan was solubilized by adding $400 \mu \mathrm{l}$ of dimethyl sulphoxide. The absorbance of each well was read at $570 \mathrm{NM}$ with immunosorbent assay (ELISA) plate reader (Hiperion MPR4, Germany). The HADSCs in 
Tab. 1.The genes and primer sequences used for real-time PCR.

\begin{tabular}{lcc}
\hline Gene & \multicolumn{1}{c}{ Primer sequences } & Size(base pair) \\
\hline Collagen II-F & CTGGTGATGATGGTGAAG & 130 \\
Collagen II-R & CCTGGATAACCTCTGTGA & \\
Sox-9-F & TCAGCAGCCAATAAGTG & 133 \\
$\begin{array}{l}\text { Sox-9-R } \\
\text { Aggrecan-F }\end{array}$ & GTGGAATGTCTTGAAGGTTA & \\
Aggrecan-R & CGGGCTTCCACCAGTGCG & 127 \\
Collagen $x-F$ & AGAATCCATCTGAGTCTCA & \\
Collagen x-R & CCTCTTACTGCTATACCTTTAC & 187 \\
$\begin{array}{l}\text { GAPDH-F } \\
\text { GAPDH-R }\end{array}$ & AAGCTCATTTCCTGGTATG & 125 \\
\hline
\end{tabular}

fibrin scaffold without any growth factor in MTT assay were likewise applied as the control group and the data was detracted from the measured values. This assay was performed in triplicate (19).

\section{Analysis of chondrogenic genes expression by real time PCR}

Expression of the hyaline cartilage-specific markers (SOX9, COLII and Aggrecan) and chondrocyte hypertrophy marker (COLX) was assessed for 14 days after the end of chondrogenic differentiation. The scaffolds were degraded within liquid nitrogen and then total RNA was extracted from all samples using Trizol in accordance with the manufacturer's protocol. Subsequently, the reverse transcription of RNA was carried out to produce a complementary DNA (cDNA) using the AccuPower RT PreMix (bioNEER, Daedeok-gu, Daejeon, Korea). Real-time PCR was completed using SYBRGreen PCR Master Mix and step one Series Software version 2.6 (Corbett Life Science, Australia), and primers for each gene were designed following the utilizing primer3 program, whose primer sequences are presented in the table 1 . The reaction began by heating to $95{ }^{\circ} \mathrm{C}$ for $15 \mathrm{~min}$., followed by 40 cycles of elongation at $59{ }^{\circ} \mathrm{C}$ for $30 \mathrm{sec}$ and denaturation at $95^{\circ} \mathrm{C}$ for $15 \mathrm{sec}$. Target gene was normalized based on GAPDH reference gene. The level of expression of each target gene was calculated using $2-\Delta \Delta \mathrm{Ct}(20)$.

\section{Histological examination}

At the 14th day, after the chondrogenic induction, samples of HADSCs that were seeded in fibrin scaffolds were collected, fixed with $10 \%$ formal saline for 24 hours, dehydrated by a graded series of eEthylalcohol, cleared in xylene and embedded in paraffin. Sections were cut at $4 \mathrm{~mm}$ and located on microscope slides. Safranin O (MERCK), toluidine blue (MERCK) and haematoxylin and eosin (H\&E; MERCK) were selected for staining. Toluidine blue and Safranin O were used to estimate the ECM components of cartilage glycosaminoglycan deposition and haematoxylin and eosin were used to reveal the other structures. Safranin O staining consists of staining the sections with Wiegert's iron haematoxylin working for $15 \mathrm{~min}$, fast green (MERCK) for $6 \mathrm{~min}$ and $1 \%$ safranin $\mathrm{O}$ for $6 \mathrm{~min}$. Subsequently, in each staining step, the sections were washed and then rinsed in absolute ethylalcohol. For Toluidine blue staining, the sections were plunge in for $5 \mathrm{~min}$. For H\&E staining, the sections were stained with Harris haematoxylin ((MERCK) for $15 \mathrm{~min}$, washed in running water, differentiated in acid alcohol $1 \%$ and then a blue stain enhancement was done by immersion in $0.05 \%$ carbonate lithium (Sigma) for $5 \mathrm{~s}$. then the sections were washed in running water and stained in Eosin-Y (MERCK)) for $3 \mathrm{~min}$. Finally, all slides were dehydrated by immersion in ethylalcohol and cleared in xylene then mounting using Microscopy Entellan (Merck) for later observation under a light microscope (Olympus BX43), the stained sections were seen (21).

\section{Immunohistochemistry assay}

Immunohistochemistry was done on the samples on 14th day. The samples were fixed in $10 \%$ formal saline for 24 hours and then embedded in paraffin and sectioned at $4 \mu \mathrm{m}$. Antigen retrieval for collagen II was done by incubation with $8 \mathrm{mg} / \mathrm{ml}$ hyaluronidase (Sigma) for 3 hours at $37{ }^{\circ} \mathrm{C}$ but antigen retrieval for collagen $\mathrm{X}$ required $2 \mathrm{mg} / \mathrm{ml}$ hyaluronidase (Sigma) for 2 hours. Furthermore, collagen X samples were treated with $1 \mathrm{mg} / \mathrm{ml}$ Pronase (Sigma). With blocking buffer, the nonspecific binding sites were blocked and sections were incubated with primary antibodies at $4{ }^{\circ} \mathrm{C}$ overnight. Monoclonal antibodies directed against human antigens were available for type II collagen (ab3092; Abcam) or type X collagen (C7974; Sigma). Sections were washed and incubated with the anti-mouse IgG secondary antibody (ab2891; Abcam) that was connected to horseradish peroxidase and was established using 3,3'- diaminobenzene (DAB) substrate kit (ab94656; Abcam) (22). To semi-quantify the immunoreactivity of type II and $X$ collagen in Kartogenin, TGF- $\beta 3$ and the control groups on day 14 , the images from each group were measured using the ImageJ software (version 1.8.0_112).

\section{Results}

\section{MTT assay}

After the use of MTT solution, the blue-black formazan crystals were seen in HADSCs, which demonstrated their metabolic activity. Applying KGN improved the proliferation and viability of HADSCs, but the evaluation of the results showed that it did not have significant differences $(\mathrm{p}>0.05)$, but in the group that was treated by TGF- $\beta 3$ decreasing of cell viability and proliferation had a significant difference $(\mathrm{p}<0.05)$ compared to the control group. These results showed that KGN in comparison with TGF- $\beta 3$ is not fatal to the cells during the 14 days (Fig. 2).
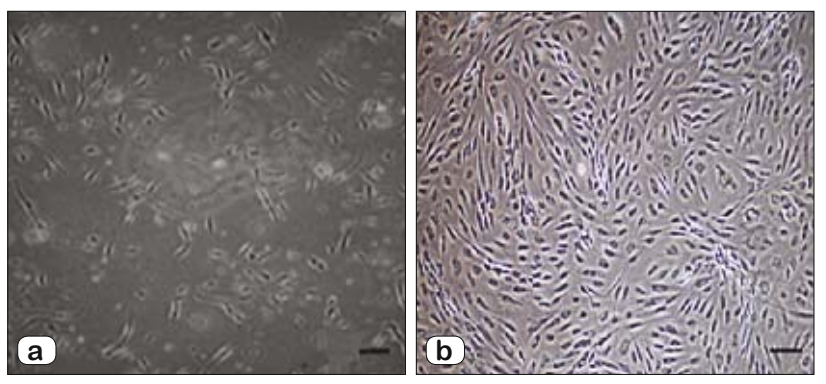

Fig. 1. Inverted microscopic image of monolayer adipose-derived stem cells at passage three (magnification $\times 40$ ), (a: 2 nd day, b: 5 th day) bar indicated $20 \mu \mathrm{m}$. 


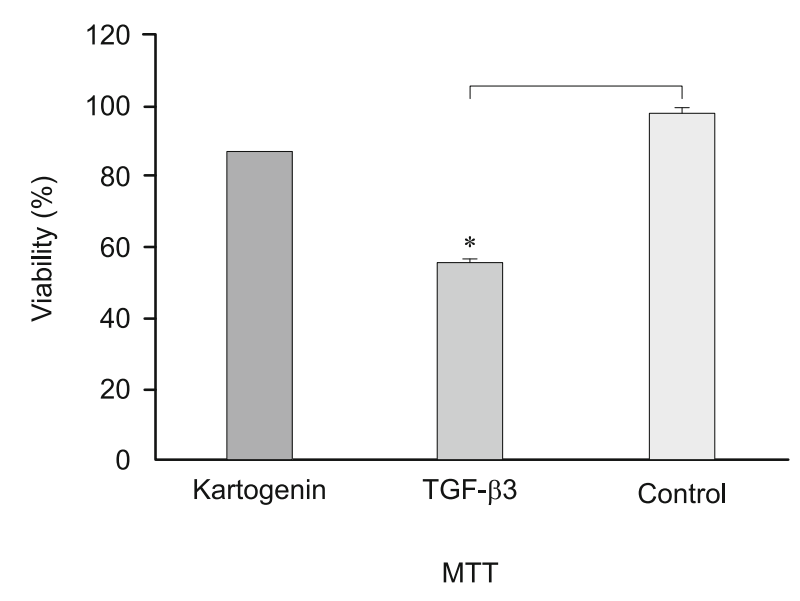

Fig. 2. Comparison of MTT assay results between their groups at 14th day. They have significant differences $(* p<0.05)$ between the group that has been treated by TGF- $\beta 3$ with the Control group.
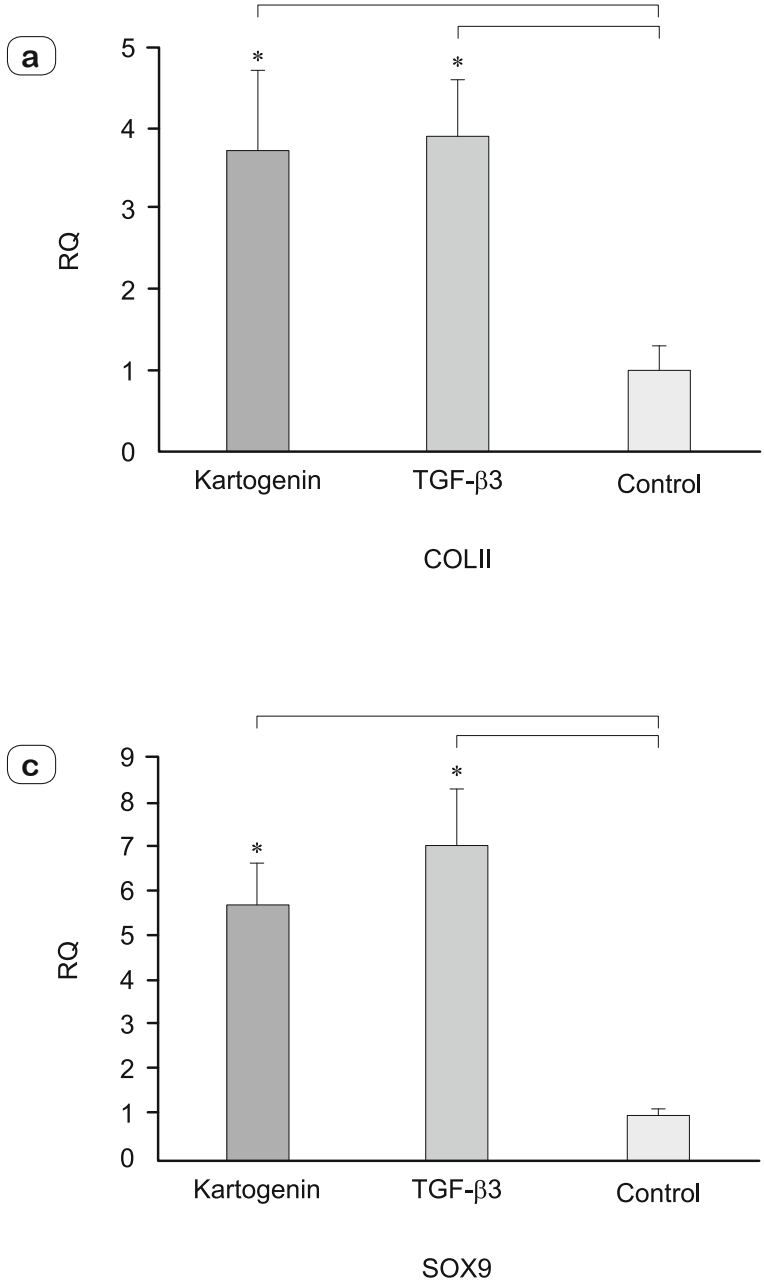

Real-time PCR

The general patterns of gene expression are briefed in the Figure 3. GAPDH was selected as the reference gene. The results of the real-time PCR indicated that COLII, AGG, and SOX9 genes expression both in KGN and TGF- $\beta$ groups were significantly higher $(\mathrm{p}<0.05)$ than in the control group, but COLX gene expression only in TGF- $\beta 3$ group was significantly higher $(\mathrm{p}<0.05)$ than in the control group (Fig. 3).

\section{Histology analysis}

The histological examination of the constructs showed the spreading of chondrocyte shape cells inside the lacuna (Fig. 4 (white arrow)) from fibrin scaffold and the deposition of extracellular matrix. Toluidine blue and safranin $\mathrm{O}$ stain the glycosaminoglycan so that is visible. The GAG deposition (Fig. 4 (black arrow)) was higher in KGN and TGF- $\beta 3$ groups than in the control group (Fig. 4). (b)

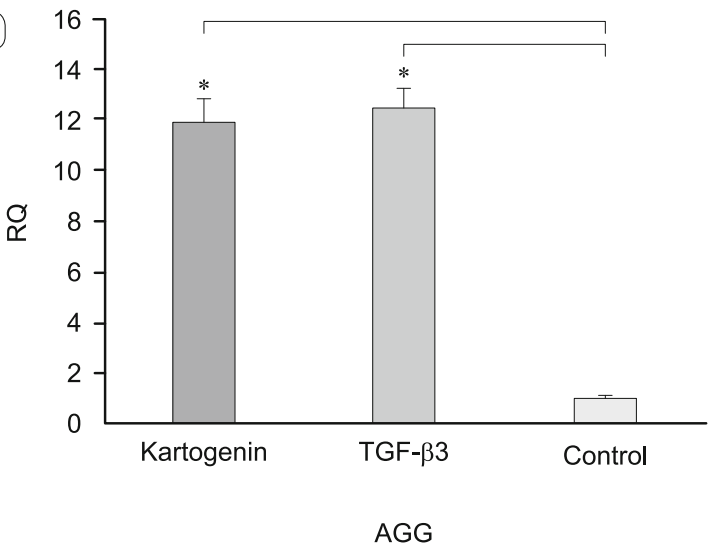

d

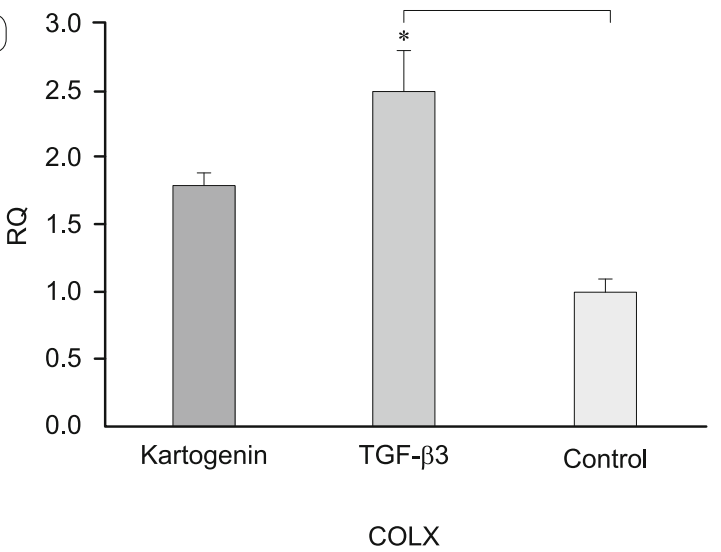

Fig. 3. Reverse transcriptase-polymerase chain reaction at the 14th day for different genes. Data presented as fold changes from the 14th day. a) Collagen type II, b) AGG, c) SOX9 and d) Collagen type X. Error bars represent standard error of the mean. The asterisk indicates that the medium condition is significantly different from Control by $(* \mathrm{p}<0.05)$. 
Immunohistochemistry analysis

The immunohistological analysis showed the distribution of collagen type II and X in the extracellular matrix in fibrin scaffold both in KGN group and in TGF- $\beta 3$ group. Localization of collagen
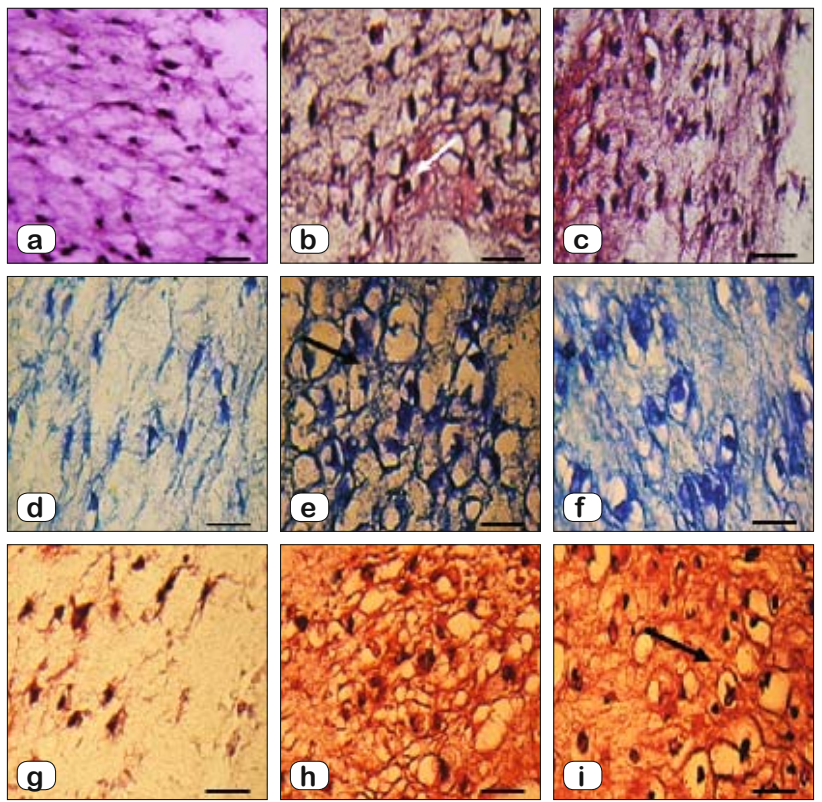

Fig. 4. Histological sections of neo-cartilage formed by Katrogenin and TGF- $\beta 3$ in fibrin scaffold cultures after 14 days as determined by $H \& E$ (a: Control, b: Kartogenin, c: TGF- $\beta 3$ ), Toluidine blue (d: Control, e: Kartogenin, f: TGF- 33 ), Safranin O (g: Control, h: Kartogenin, i: TGF- $\beta 3$ ), magnification $\times 40$, bar indicated $20 \mu \mathrm{m}$.

(A)
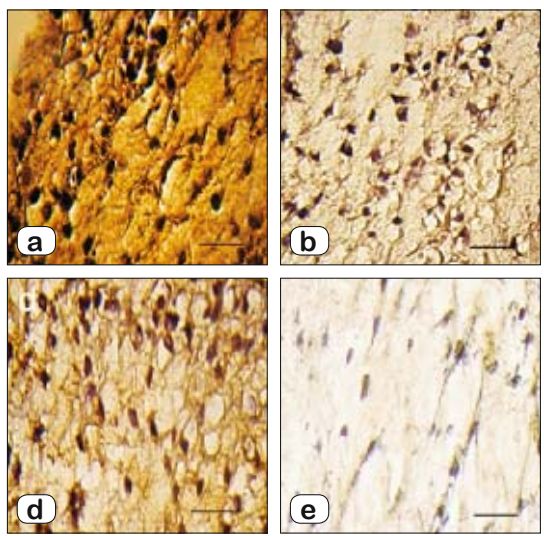

Fig. 5. A: Immunohistochemically sections of neo-cartilage formed by KGN and TGF- $\beta 3$ in fibrin scaffold cultures after 14 days. Immunostained with anti-type II collagen antibodies (a: Kartogenin, c: TGF- $\beta 3$ and e: Control) and anti-type $X$ collagen antibodies (b: Kartogenin, d: TGF- $\beta 3$ and f: Control) in vitro culture, magnification $\times 40$, bar indicated $20 \mu \mathrm{m}$. B. a: color intensity demonstrated the high deposition of type II collagen protein in KGN and TGF- $\beta 3$ treatment groups compared to the control group. The asterisk indicates that the medium condition is significantly different from the control group by (* $p<0.05)$. B.b: color intensity demonstrated the high deposition of type $X$ collagen protein only in TGF- $\beta 3$ treatment group compared to the control group. The asterisk indicates that the medium condition is significantly different from the control group by $\left({ }^{*} p<0.05\right)$. type II in pre-cellular matrix confirmed that HADSCs chondrogenic differentiation has happened. The average percentage of type II collagen positive area (Fig. 5) were found to be significantly higher in KGN and TGF- $\beta 3$ groups than in the control $(\mathrm{p}<0.05)$. The collagen type $\mathrm{X}$, a cartilage hypertrophic phenotypic marker was detected in the collagenous matrix of all groups, but in the group that was treated by TGF- $\beta 3$ deposition, the collagen type $\mathrm{X}$ was higher than in KGN and in the control groups, significantly $(\mathrm{p}<0.05)$ (Fig. 5).

\section{Discussion}

Tissue engineering was the best choice that can be used to develop healing methods to fight the progression of OA and to produce cartilage to treat the damaged tissue. Ensuring that these constructs keep the same functional properties as native cartilage tissue is very important. However, tissue engineering techniques have been used to produce cartilage-like tissues (23). Appropriate cell, scaffold and growth factors were principal components of tissue engineering (24). In the current study, for the first time, the effect of the small molecule, kartogenin (KGN) was examined on chondrogenic induction process on human adipose-derived stem cells (hADSc) in fibrin scaffolds. Fibrin scaffold was used as a template for chondrogenic induction. Previous studies demonstrated that hydrogels scaffold such as fibrin is non-toxic composition and in contrast to the synthetic scaffolds could absolutely capture the cells, preventing cell damage and thus has a high delivery efficiency (25). Dongguan Shi, et al reported, which

B
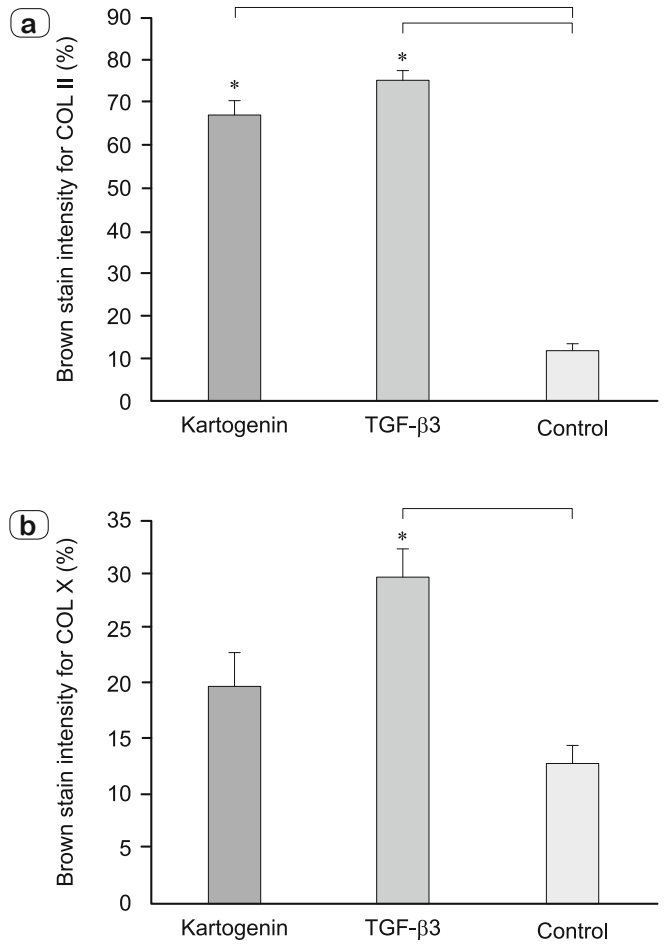
591-597

of the sustained-release properties of hydrogel scaffold with KGN enhanced the treatment of the defects and the generation of hyaline cartilage (26). On the other hand, the differentiation phase of chondrogenesis progress, cell-matrix interactions facilitated by bindings of cells to extracellular matrix fibre, increased cell proliferation and ECM remodelling (12). Our evidence suggested that the use of fibrin scaffold for chondrogenesis was suitable. The use of histology, immunohistochemistry and real-time PCR demonstrated that KGN like TGF- $\beta 3$ had a significant effect on differentiation of HADSc to chondrocyte. Many studies reported that TGF- $\beta 3$ had a strong impact on chondrogenic induction in stem cells (11) but there was no evidence about the effect of KGN on chondrogenic induction in HADSc. Our results showed that KGN could induce chondrogenic differentiation by surging and accumulation of glycosaminoglycan in HADSc cultured with 100 NM KGN concentrations (Fig. 3). Ting Yuan, Et al, likewise reported that $\mathrm{KGN}$ induced chondrogenic differentiation in rat tendons in vivo (27). Also, KGN by increasing the accumulation of glycosaminoglycan in tendon stem/progenitor cells (TSCs) culture can bring chondrogenic differentiation (27). In 2012, KGN was identified as a small heterocyclic molecule that could stimulate chondrogenesis in MSCs (16). Johnson and colleagues declared that KGN enhanced the gene expression of aggrecan and type II collagen protein in MSCs (16). KGN blocks the interaction between $\mathrm{CBFb}$ and $\mathrm{FC}-1$ and also $\mathrm{CBFb}$ is the regulatory subunit of the heterodimeric core binding factor transcription complex (17). The other subunits are one of runt-related transcription factor (RUNX) family members that contain RUNX1, RUNX2 and RUNX3 (28). Likely, this mechanism as well applies to the developmental progressions that these genes are expressed in limb pre-skeletal mesenchymal cells (28) and RUNX1 has been revealed to stimulate their differentiation (29). It seems that KGN, through the RUNX1 pathway, plays a critical role in chondrogenesis and chondrocyte proliferation. Overexpression of collagen II gene and consequently an increase of the protein of collagen II suggests that KGN like other growth factors such as: TGF betas, BMPs or IGFs is able to activate the chondrogenic induction in HADSc. Previous studies showed that phosphorylation of Smad $2 / 3$ and Smad $1 / 5 / 8$ is responsible for TGF- $\beta$ family(30). Many studies showed that activating the pathway of Smad $1 / 5 / 8$ performance upstream of RUNX2 is the basis of hypertrophy and terminal differentiation of chondrocytes (30), but KGN only increases the expression of Smad 2/3 and inhibited the expression of Smad 1/5/8 (30). Our evidences suggested that the expression of COL X that indicated the rate of chondrocyte hypertrophy significantly decreased compered to TGF- $\beta 3$ group. KGN may help refresh articular cartilage by shifting the differentiation balance from late differentiation (hypertrophy) towards early differentiation (chondrogenesis). Despite numerous reports about the effect of KGN on the chondrogenic induction, other studies showed that KGN could not induce chondrogenesis alone, but along with the other growth factors could be effective (31). But in this study, we did observe that $\mathrm{KGN}$, alone as a growth factor was capable of inducing chondrogenesis in human adipose-derived stem cells. However, more research is needed in this area.

\section{Conclusion}

Our investigation demonstrated that compared to TGF- $\beta 3$, a small molecule, KGN, could promote chondrogenic differentiation of HADSCs and enhance the accumulation of glycosaminoglycan along with a decline of type X collagen as well. Our evidences suggested that KGN acted as an inducible factor in the field of cartilage repair.

\section{References}

1. Casasnovas Ortega N. Developing osteoarthritis treatments through cartilage tissue engineering and molecular imaging. Massachusetts Institute of Technology, 2012.

2. Xie L, Wang X, Zhou S. Mesenchymal Stem Cells and Cartilage Regeneration in Traumatic and Osteoarthritic Cartilage Defects. Enliven: J Stem Cells Regen Med 2014; 1 (1): 2.

3. Diekman BO, Guilak F. Stem cell-based therapies for osteoarthritis: challenges and opportunities. Curr Opinion Rheumatol 2014; 25 (1): 119.

4. Hamid AA, Idrus RBH, Saim AB, Sathappan S, Chua KH. Characterization of human adipose-derived stem cells and expression of chondrogenic genes during induction of cartilage differentiation. Clinics 2012; 67 (2): 099-106.

5. Strem BM, Hedrick MH. The growing importance of fat in regenerative medicine. Trends Biotechnol 2005; 23 (2): 64-66.

6. Ahmed TA, Dare EV, Hincke M. Fibrin: a versatile scaffold for tissue engineering applications. Tissue Engineering Part B: Reviews 2008; 14 (2): 199-215.

7. Fenger-Eriksen C, Ingerslev J, Sørensen B. Fibrinogen concentratea potential universal hemostatic agent. Expert Opinion Biol Ther 2009; 9 (10): 1325-1333.

8. Geer DJ, Swartz DD, Andreadis ST. Fibrin promotes migration in a three-dimensional in vitro model of wound regeneration. Tissue Engineering 2002; 8 (5): 787-798.

9. Danišovič L', Varga I, Polák Š. Growth factors and chondrogenic differentiation of mesenchymal stem cells. Tissue Cell 2012; 44 (2): 69-73.

10. Park KH, Na K. Effect of growth factors on chondrogenic differentiation of rabbit mesenchymal cells embedded in injectable hydrogels. J Biosci Bioengineering 2008; 106 (1): 74-79.

11. Augustyniak E, Trzeciak T, Richter M, Kaczmarczyk J, Suchorska W. The role of growth factors in stem cell-directed chondrogenesis: a real hope for damaged cartilage regeneration. Internat Orthopaed 2015; 39 (5): 995-1003.

12. Hidaka C, Goldring MB. Regulatory mechanisms of chondrogenesis and implications for understanding articular cartilage homeostasis. Curr Rheumatol Rev 2008; 4 (3): 136-147.

13. Furumatsu T, Ozaki T, Asahara H. Smad 3 activates the Sox9-dependent transcription on chromatin. Internat J Biochem Cell Biol 2009; 41 (5): 1198-1204.

14. Finnson KW, Chi Y, Bou-Gharios G, Leask A, Philip A. TGF-b signaling in cartilage homeostasis and osteoarthritis. Front Biosci (Schol Ed) $2012 ; 4: 251-268$.

15. Goldring MB, Goldring SR. Articular cartilage and subchondral bone in the pathogenesis of osteoarthritis. Ann NY Acad Sci 2010; 1192 (1): $230-237$. 
16. Johnson K, Zhu S, Tremblay MS, Payette JN, Wang J, Bouchez LC, Meeusen S, Althage A, Cho CY, Wu X. A stem cell-based approach to cartilage repair. Science 2012; 336 (6082): 717-721.

17. Yoshida N, Ogata T, Tanabe K, Li S, Nakazato M, Kohu K, Takafuta T, Shapiro S, Ohta Y, Satake M. Filamin A-bound PEBP2 $\beta / C B F \beta$ is retained in the cytoplasm and prevented from functioning as a partner of the Runx1 transcription factor. Mol Cell Biol 2005; 25 (3): 1003-1012.

18. Mardani M, Hashemibeni B, Ansar MM, Esfahani Z, Hamid S, Kazemi M, Goharian V, Esmaeili N, Esfandiary E. Comparison between chondrogenic markers of differentiated chondrocytes from adipose derived stem cells and articular chondrocytes in vitro. Iran J Basic Med Sci 2013; 16 (6): 763-771.

19. Esfandiari E, Roshankhah S, Mardani M, Hashemibeni B, Naghsh E, Kazemi M, Salahshoor M. The effect of high frequency electric field on enhancement of chondrogenesis in human adipose-derived stem cells. Iran J Basic Med Sci 2014;17 (8): 571.

20. Valiani A, Hashemibeni B, Esfandiary E, Ansar MM, Kazemi M, Esmaeili N. Study of carbon nano-tubes effects on the chondrogenesis of human adipose derived stem cells in alginate scaffold. Internat J Prevent Med 2014; 5 (7): 825.

21. Talakoob S, Joghataei MT, Parivar K, Bananej M, Sanadgol N. Capability of Cartilage Extract to In Vitro Differentiation of Rat Mesenchymal Stem Cells (MSCs) to Chondrocyte Lineage. Internat J Mol Cell Med 2015; 4 (1): 9.

22. Kabiri A, Esfandiari E, Esmaeili A, Hashemibeni B, Pourazar A, Mardani M. Platelet-rich plasma application in chondrogenesis. Adv Biomed Res 3, 2014.

23. Varga I, Zamborský R, Böhmer D. The tissue engineering of articular cartilage: cells, scaffolds and stimulating factors. Exp Biol Med 2012; 237 (1): 10-17.
24. Vinatier C, Guicheux J. Cartilage tissue engineering: From biomaterials and stem cells to osteoarthritis treatments. Ann Phys Rehab Med 2016; 59 (3): 139-144.

25. Thomson KS, Dupras SK, Murry CE, Scatena M, Regnier M. Proangiogenic microtemplated fibrin scaffolds containing aprotinin promote improved wound healing responses. Angiogenesis 2014; 17 (1): 195-205.

26. Shi D, Xu X, Ye Y, Song K, Cheng Y, Di J, Hu Q, Li J, Ju H, Jiang Q. Photo-cross-linked scaffold with kartogenin-encapsulated nanoparticles for cartilage regeneration. ACS nano 2016; 10 (1): 1292-1299.

27. Yuan T, Zhang J, Zhao G, Zhou Y, Zhang C-Q, Wang JH. Creating an Animal Model of Tendinopathy by Inducing Chondrogenic Differentiation with Kartogenin. PloS one 2016; 11 (2): e0148557.

28. Adya N, Castilla L, Liu P. Function of CBF $\beta /$ Bro proteins. Semin Cell Develop Biol 2000; 5: 361-368.

29. Wang Y, Belflower RM, Dong YF, Schwarz EM, O'Keefe RJ, Drissi H. Runx1/AML1/Cbfa2 mediates onset of mesenchymal cell differentiation toward chondrogenesis. J Bone Miner Res 2005; 20 (9): 1624-1636.

30. Wang J, Zhou J, Zhang N, Zhang X, Li Q. A heterocyclic molecule kartogenin induces collagen synthesis of human dermal fibroblasts by activating the smad4/smad5 pathway. Biochem Biophys Res Comm 2014; 450 (1): 568-574.

31. Liu C, Ma X, Li T, Zhang Q. Kartogenin, transforming growth factor- $\beta 1$ and bone morphogenetic protein-7 coordinately enhance lubricin accumulation in bone-derived mesenchymal stem cells. Cell Biol Internat 2015; 39 (9): 1026-1035.

Received May 27, 2017. Accepted June 19, 2017. 